\title{
A COMMUTATOR THEOREM AND WEIGHTED BMO
}

BY

\author{
STEVEN BLOOM
}

\begin{abstract}
The main result of this paper is a commutator theorem: If $\mu$ and $\lambda$ are $A_{p}$ weights, then the commutator $H, M_{b}$ is a bounded operator from $L^{p}(\mu)$ into $L^{p}(\lambda)$

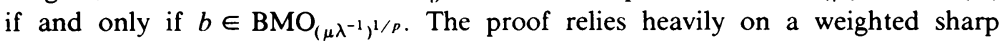
function theorem. Along the way, several other applications of this theorem are derived, including a doubly-weighted $L^{p}$ estimate for BMO. Finally, the commutator theorem is used to obtain vector-valued weighted norm inequalities for the Hilbert transform.
\end{abstract}

I. Introduction. In the last decade, there have been several major results involving weighted norm inequalities for the conjugate operator $f \rightarrow H f$, given for trigonometric polynomials $f=\sum c_{n} r^{|n|} e^{i n \theta}$ by

$$
H f(\theta)=i \sum_{n \leqslant-1} c_{n} r^{|n|} e^{i n \theta}-i \sum_{n \geqslant 1} c_{n} r^{n} e^{i n \theta} .
$$

$H$ may also be viewed as a convolution with conjugate Poisson kernel $Q$,

$$
Q(x)=(\sin x) / 1-\cos x .
$$

The major results involved the class of $A_{p}$ weights and their logarithms.

Definition 1.1. A nonnegative function $w$ is in the class $\left(A_{p}\right)$, for $1<p<\infty$, if there exists a constant $C$ so that, for $1 / p+1 / q=1$ and all intervals $I$ contained in the boundary of the unit circle, we have

$$
\frac{1}{|I|} \int_{I} w(x) d x\left(\frac{1}{|I|} \sum_{I} w^{-q / p}(x) d x\right)^{p-1} \leqslant C,
$$

(where $|I|$ denotes the measure of the interval $I$ ).

Two useful properties of weights are the $A_{\infty}$ condition: $w$ is in the class $\left(A_{\infty}\right)$ if there exist constants $C$ and $\delta>0$ so that, for each interval $I$ and measurable set $E \subseteq I$, we have

$$
\left(\int_{E} w\right) /\left(\int_{I} w\right) \leqslant C\left(\frac{|E|^{\delta}}{|I|}\right)
$$

and the Reverse Hölder condition: There exist constants $C$ and $\delta>0$ such that, for all intervals $I$,

$$
\left(\frac{1}{|I|} \int_{I} w^{1+\delta} d x\right)^{1 /(1+\delta)} \leqslant \frac{C}{|I|} \int_{I} w d x .
$$

Muckenhoupt $[7,8]$ has shown that $A_{p}$ for some $p>1, A_{\infty}$, and Reverse Hölder are all equivalent.

Received by the editors June 27, 1984.

1980 Mathematics Subject Classification. Primary 42A50; Secondary 42B20, 46E40. 
The major weighted norm inequality for the conjugate operator was proven by Hunt, Muckenhoupt, and Wheeden [5].

THEOREM 1.2. $H$ is a bounded operator on the weighted $L^{p}$-space $L^{p}(w)$ if and only if $w \in\left(A_{p}\right)$.

There is one more approach to the conjugate operator that has proved fruitful, via commutators and the class BMO. Let $M_{b}$ denote multiplication by the function $b$. The commutator $\left[H, M_{b}\right]$ is the operator taking $f \rightarrow H(b f)-b(H f)$. The bounded operators of this form comprise the dual space of $H^{1}$, so that, by Fefferman's Theorem, $\left[H, M_{h}\right]$ is a bounded operator of $L^{2}$ if and only if $b \in \mathrm{BMO}$, the class of functions of bounded mean oscillation:

$$
\sup _{I} \frac{1}{|I|} \int_{I}\left|b(x)-\frac{1}{|I|} \int_{I} b(t) d t\right| d x<\infty .
$$

(See Coifman, Rochberg, and Weiss [3].)

This theory is linked to the weighted norm inequalities in the following way: If $b \in \mathrm{BMO}$, by the John-Nirenberg Theorem, $\exp (t b) \in\left(A_{p}\right)$ for $t$ sufficiently small and $p>1$ fixed. Thus $H$ is a bounded operator on $L^{p}\left(e^{t b}\right)$.

Conversely, if $H$ is a bounded operator on $L^{2}\left(e^{b}\right)$, then the operators $T_{z}=e^{z b} H e^{-z b}$ are bounded operators on $L^{2}$ for $|z| \leqslant \frac{1}{2}$, as is $\left.(d / d t) T_{t}\right|_{t=0}=\left[H, M_{b}\right]$, so that $b \in \mathrm{BMO}$.

In this paper, we extend this work to settings involving multiple weights. In §II, we present a weighted sharp function theorem, which plays a key role in the later analysis. In §III, we present some simple applications of this theorem. In $\S I V$, we present the Commutator Theorem: If $\mu$ and $\lambda$ are $A_{p}$ weights, then the commutator $\left[H, M_{b}\right]$ is a bounded map from $L^{p}(\mu)$ into $L^{p}(\lambda)$ if and only if $b$ is in an appropriate weighted BMO space. And in $\S \mathrm{V}$, we present a vector-valued version of Theorem 1.2.

II. The Sharp Function Theorem. A measure $\nu$ is a doubling measure if there exists a constant $C$ such that, for any intervals $I$ and $J$ with $|J|=2|I|$, we have $\nu(J) \leqslant$ $C \nu(I)$.

For example, if $w \in\left(A_{p}\right)$, then the measure $w d x$ is a doubling measure.

The unit circle will be denoted by $T$.

Let $\nu$ be a doubling measure and $u$ a nonnegative weight. Then $u$ induces a measure, which we also call $u$, given by

$$
u(E)=\int_{E} u d \nu
$$

$u$ is in the class $A_{\infty}(d \nu)$ if there exist constants $C$ and $\delta>0$ for which

$$
u(E) / u(I) \leqslant C(\nu(E) / \nu(I))^{\delta}
$$

for all intervals $I$ and measurable sets $E \subseteq I$. This measure $u$ is also a doubling measure. The average of a function $f$ over an interval $I$ will be denoted by $f_{I}$,

$$
f_{I}=\frac{I}{\nu(I)} \int_{I} f d \nu
$$


or sometimes $f_{I, d \nu}$ if the doubling measure is in doubt. The maximal function and the sharp function (relative to $\nu$ ) are given by

$$
f^{*}(x)=\sup \left\{|f|_{I}: x \in I\right\}, \text { and } f^{\#}(x)=\sup \left\{\left|f-f_{I}\right|_{I}: x \in I\right\} .
$$

Finally, $L^{p}(u)$ will denote the $L^{p}$-space on the circle with norm

$$
\|f\|_{L^{p}(u)}=\left(\frac{1}{u(T)} \int_{T}|f|^{p} u d \nu\right)^{1 / p} .
$$

Theorem 2.1 (The Sharp Function TheORem). Let $f \in L^{1}(d v), 1<p<\infty$, and $f^{\#} \in L^{p}(u)$, for some $u \in A_{\infty}(d v)$. Then $f \in L^{p}(u)$, with

$$
\left\|f-f_{T}\right\|_{L^{p}(u)} \leqslant C_{p}\left\|f^{\#}\right\|_{L^{p}(u)} .
$$

An unweighted version of this theorem, with $u=1$ and $\nu$ Lebesgue measure, was given by Fefferman and Stein. Extending their proof to the present setting is straightforward and we omit the details (which can be found in [1]; see also [11]).

This theorem could have been proven for functions restricted to any interval $I \subseteq T$. Let $f^{\#, I}$ denote the sharp function restricted to $I$,

$$
f^{\#, I}(x)=\sup \left\{\frac{1}{\nu(J)} \int_{J}\left|f-f_{J}\right| d \nu: x \in J \subseteq I\right\} .
$$

In this case, we would find

Corollary 2.2. Let $f \in L^{1}(d \nu), u \in A_{\infty}(d \nu)$, and $1<p<\infty$. If $f^{\#} \in L^{p}(u d \nu)$, then so is $f$, and for any interval $I \subseteq T$, we have

$$
\int_{I}\left|f-f_{I}\right|^{p} u d \nu \leqslant C_{p} \int_{I}\left(f^{\#, I}\right)^{p} u d \nu \leqslant C_{p} \int_{I}\left(f^{\#}\right)^{p} u d \nu,
$$

where $C_{p}$ does not depend on I or $f$.

III. Applications of the Sharp Function Theorem. Our applications involve $L^{p}$ estimates for some nonstandard weighted BMO classes. The first class we will consider is the doubly-weighted BMO class. Let $u$ and $v$ be weights, and suppose that

$$
\inf _{c_{I}} \int_{I}\left|f-c_{I}\right| u \leqslant C \int_{I} v \quad \text { for } c_{I} \text { constants. }
$$

Is there an $L^{p}$ version of this for any $p>1$ ? When $u$ and $v$ are 1 , this is standard BMO, and the John-Nirenberg Theorem gives the $L^{p}$ estimate

$$
\inf _{c_{I}} \int_{I}\left|f-c_{I}\right|^{p} \leqslant C|I|
$$

for any $1 \leqslant p<\infty$. The doubly-weighted version of this follows.

Theorem 3.1 (The Doubly - Weighted BMO Theorem). Suppose that $u^{-1}$ and $v^{-1}$ are in $\left(A_{p}\right)$ for some $p<2$, and that $1 / p+1 / q=1$. If

$$
\inf _{c_{I}} \int_{I}\left|f-c_{I}\right| u \leqslant C \int_{I} v \text { for all intervals } I,
$$


then

$$
\inf _{c_{I}} \frac{1}{|I|} \int_{I}\left|f-c_{I}\right|^{q / p} u^{q / p} \leqslant K\left(\frac{1}{|I|} \int_{I} v\right)^{q / p} .
$$

Proof. We will apply the Sharp Function Theorem with the measure $\nu=u d x$. Define $f^{\#}, f^{*}$, and $f_{I}$ with respect to $u d x$. Suppose that (1) holds for some constant $c_{I}$. Then we can take $c_{I}=f_{I}$ without losing more than a factor of 2 . Now fix $x$ and let $I$ contain $x$. Then

$$
\frac{1}{u(I)} \int_{I}\left|f-f_{I}\right| u \leqslant \frac{c}{u(I)} \int_{I} v=\frac{c}{u(I)} \int_{I} v u^{-1} u \leqslant C\left(v u^{-1}\right)^{*}(x),
$$

and hence, $f^{\#}(x) \leqslant C\left(v u^{-1}\right)^{*}(x)$. Now fix an interval $I$. By Corollary 2.2,

$$
\begin{aligned}
\int_{I}\left|f-f_{I}\right|^{q / p} u^{q / p} d x & =\int_{I}\left|f-f_{I}\right|^{q / p} u^{q / p-1} u d x \\
& \leqslant C_{p} \int_{I}\left(f^{\#}\right)^{q / p} u^{q / p-1} u d x \\
& \leqslant C \cdot C_{p} \int_{I}\left(v u^{-1}\right)^{* q / p} u^{q / p-1} u d x
\end{aligned}
$$

We claim that

$$
u^{q / p-1} \in A_{q / p}(u d x) .
$$

Given (3), Muckenhoupt's Theorem implies

$$
\begin{aligned}
\frac{1}{|I|} \int_{I}\left|f-f_{I}\right|^{q / p} u^{q / p} d x & \leqslant C^{\prime} \frac{1}{|I|} \int_{I}\left(v u^{-1}\right)^{q / p} u^{q / p} d x \\
& =C^{\prime} \frac{1}{|I|} \int_{I} v^{q / p} d x \\
& \leqslant K\left(\frac{1}{|I|} \int_{I} v^{-1}\right)^{-q / p} \text { as } v^{-1} \in\left(A_{p}\right) \\
& \leqslant K\left(\frac{1}{|I|} \int_{I} v\right)^{q / p}, \quad \text { by Cauchy-Schwarz. }
\end{aligned}
$$

So we must show (3). For this we must bound

$$
\begin{aligned}
& \frac{1}{u(I)} \int_{I} u^{q / p}\left[\frac{1}{u(I)} \int_{I}\left(u^{q / p-1}\right)^{-1 /(q / p-1)} u\right]^{q /(p-1)} \\
& =\frac{1}{u(I)} \int_{I} u^{q / p}\left(\frac{1}{u(I)} \int_{I} u^{-1} u\right)^{q / p-1}=\left(\frac{1}{u(I)}\right)^{q / p}|I|^{q / p} \frac{1}{|I|} \int_{I} u^{q / p} \\
& =\left(\frac{1}{|I|} \int_{I} u\right)^{-q / p} \frac{1}{|I|} \int_{I} u^{q / p} \leqslant\left(\frac{1}{|I|} \int_{I} u^{-1}\right)^{q / p}\left(\frac{1}{|I|} \int_{I} u^{q / p}\right)
\end{aligned}
$$

and this is bounded, since $u^{-1} \in\left(A_{p}\right)$. 
For our other application, we will strengthen a Lipschitz type theorem of Lotkowski and Wheeden [6].

THEOREM 3.2. Let $F$ be a nonnegative function of sets, for which, if $I \subseteq J$, then $F(I) \leqslant C F(J)$. Let $\mu$ be a doubling measure, and let $g^{-1} \in A_{p}(g d \mu), 1<p<\infty$. If for each interval $I$,

$$
\int_{I}\left|f-f_{I}\right| g d \mu \leqslant C F(I) \mu(I),
$$

where $f_{I}=f_{I, g d \mu}$ is the average with respect to $g d \mu$, then

$$
\int_{I}\left(\left|f-f_{I}\right| g\right)^{q} d \mu \leqslant C F(I)^{q} \mu(I), \quad \frac{1}{p}+\frac{1}{q}=1 .
$$

(Note: Here, and throughout, $C$ will denote a universal constant, not necessarily the same at successive appearances.)

Lotkowski and Wheeden also assumed the existence of constants $1<\alpha<\beta$ for which $\alpha F(I) \leqslant F(2 I) \leqslant \beta F(I)$. In particular, the restriction $\alpha>1$ ruled out the function $F \equiv 1$.

Proof of Theorem 3.2. Let $\nu$ be the measure $g d \mu$, and define $f_{I}, f^{*}$, and $f^{\#}$ with respect to $\nu$. Fix $I$ and let $x \in J \subseteq I$. Then

$$
\int_{J}\left|f-f_{J}\right| d \nu \leqslant C F(J) \mu(J) \leqslant C F(I) \mu(J) .
$$

Hence,

$$
\frac{1}{\nu(J)} \int_{J}\left|f-f_{J}\right| d \nu \leqslant C F(I) \frac{1}{\nu(J)} \int_{J} g^{-1} g d \mu \leqslant C F(I)\left(g^{-1}\right)^{*}(x),
$$

and so, taking supremums over $J \subseteq I$, we have $f^{\#, I}(x) \leqslant C F(I)\left(g^{-1}\right)^{*}(x)$. By Corollary 2.2,

$$
\begin{aligned}
\int_{I}\left|f-f_{I}\right|^{q} g^{q-1} d \nu & \leqslant C \int_{I}\left(f^{\# . I}\right)^{q} g^{q-1} d \nu \\
& \leqslant C F(I)^{q} \int_{I}\left(g^{-1}\right)^{* q} g^{q-1} d \nu .
\end{aligned}
$$

But $g^{q-1} \in A_{q}(d \nu)$, as

$$
\begin{aligned}
& \frac{1}{\nu(I)} \int_{I} g^{q-1} d \nu\left(\frac{1}{\nu(I)} \int_{I} g^{-1} d \nu\right)^{q-1} \\
& \quad=\frac{1}{\nu(I)} \int_{I}\left(g^{-1}\right)^{1 /(p-1)} d \nu\left(\frac{1}{\nu(I)} \int_{I} g^{-1} d \nu\right)^{q-1}
\end{aligned}
$$

which is bounded by the hypothesis $g^{-1} \in A_{p}(d \nu)$. Applying Muckenhoupt's Theorem, we conclude

$$
\int_{I}\left|f-f_{I}\right|^{q} g^{q} d \mu \leqslant C F(I)^{q} \int_{I}\left(g^{-1}\right)^{q} g^{q} d \mu=C F(I)^{q} \mu(I) .
$$




\section{The Commutator Theorem.}

Definition 4.1. Let $w$ be an $A_{\infty}$ weight and $b$ an $L^{1}$ function. Then $b$ is in the weighted $\mathrm{BMO}$ class $\mathrm{BMO}_{w}$ provided

$$
\sup _{I} \frac{1}{w(I)} \int_{I}\left|b-b_{I}\right|<\infty \quad\left(\text { here } b_{I}=b_{I, d x}\right) .
$$

The main result of this paper is the following:

Theorem 4.2 (The Commutator Theorem). Let $\mu, \lambda \in\left(A_{p}\right)$ and put $\nu=$ $\left(\mu \lambda^{-1}\right)^{1 / p}$, for some $1<p<\infty$, and suppose that $b \in L^{1}$. Then

(i) If $b \in \mathrm{BMO}_{v}$, the commutator $\left[H, M_{b}\right]$ is a bounded map from $L^{p}(\mu)$ into $L^{p}(\lambda)$, with

$$
\int\left|\left[H, M_{b}\right] f\right|^{p} \lambda \leqslant C \int|f|^{p} \mu .
$$

(ii) Conversely, if $\left[H, M_{b}\right]: L^{p}(\mu) \rightarrow L^{p}(\lambda)$ is bounded, then $b \in \mathrm{BMO}_{\nu}$.

To prove part (i), we will need a series of lemmas. Throughout, $\mu$ and $\lambda$ will be in $\left(A_{p}\right), \nu=\left(\mu \lambda^{-1}\right)^{1 / p}$, and $b \in \mathrm{BMO}_{\nu}$. An exponent with a prime will denote the conjugate exponent, so $1 / p+1 / p^{\prime}=1$.

Lemma 4.3. There exists an $\varepsilon>0$ so that, for all $1 \leqslant r \leqslant p^{\prime}+\varepsilon$,

$$
\frac{1}{|I|} \int_{I}\left|b-b_{I}\right|^{r} \mu^{-r / p} \leqslant C\left(\frac{1}{|I|} \int_{I} \lambda^{-1 / p}\right)^{r} \text { for each interval } I \text {. }
$$

Proof. It will suffice to show this for some $r>p^{\prime}$. Smaller values of $r$ follow from Hölder's Inequality.

Choose $r$ so that Reverse Hölder holds for the weights $\mu^{-p^{\prime} / p}$ and $\lambda^{-p^{\prime} / p}$ with exponent $1+\delta=r / p^{\prime}$. Fix $I$ and let $x \in I$. If $J$ contains $x$, then

$$
\frac{1}{|I|} \int_{J}\left|b-b_{J}\right| \leqslant C \frac{1}{|J|} \int_{J} \nu \leqslant C \nu^{*}(x),
$$

and hence $b^{\#}(x) \leqslant C \nu^{*}(x)$. By Corollary 2.2 ,

$$
\int\left|b-b_{I}\right|^{r} \mu^{-r / p} \leqslant C \int_{I}\left(b^{\#}\right)^{r} \mu^{-r / p} \leqslant C \int_{I}\left(\nu^{*}\right)^{r} \mu^{-r / p} .
$$

But

$$
\begin{aligned}
\frac{1}{|J|} \int_{J} \mu^{-r / p}\left(\frac{1}{|J|} \int_{J} \mu^{r^{1 / p}}\right)^{r / r^{\prime}} & \leqslant \frac{1}{|J|} \int_{J} \mu^{-r / p}\left(\frac{1}{|J|} \int_{J} \mu\right)^{r / p}, \\
& \leqslant C\left(\frac{1}{|J|} \int_{J} \mu^{-p^{\prime} / p}\right)^{r / p^{\prime}}\left(\frac{1}{|J|} \int_{J} \mu\right)^{r / p}, \\
& \leqslant C \text { by }\left(A_{p}\right) .
\end{aligned}
$$


Thus, $\mu^{-r / p} \in\left(A_{r}\right)$ and Muckenhoupt's Theorem applies. So

$$
\int_{I}\left|b-b_{I}\right|^{r} \mu^{-r / p} \leqslant C \int_{I} \nu^{r} \mu^{-r / p}=C \int_{I} \lambda^{-r / p} .
$$

Similarly, $\lambda^{-r / p} \in\left(A_{r}\right)$, and

$$
\frac{1}{|I|} \int_{I} \lambda^{-r / p}\left(\frac{1}{|I|} \int_{I} \lambda\right)^{r / p} \leqslant C
$$

so that

$$
\frac{1}{|I|} \int_{I} \lambda^{-r / p} \leqslant C\left(\frac{1}{|I|} \int_{I} \lambda\right)^{-r / p} .
$$

But by Cauchy-Schwartz,

$$
1 \leqslant \frac{1}{|I|} \int_{I} \lambda^{1 / p} \frac{1}{|I|} \int_{I} \lambda^{-1 / p}
$$

so that

$$
\begin{aligned}
\frac{1}{|I|} \int\left|b-b_{I}\right|^{r} \mu^{-r / p} & \leqslant C\left(\frac{1}{|I|} \int_{I} \lambda\right)^{-r / p} \leqslant C\left(\frac{1}{|I|} \int_{I} \lambda^{1 / p}\right)^{-r}, \text { by Hölder's } \\
& \leqslant C\left(\frac{1}{|I|} \int_{I} \lambda^{-1 / p}\right)^{r}
\end{aligned}
$$

We will need some further notation. $q$ will be a number near $p$ but less than $p$. Let $r \geqslant 1$ and $w$ a weight. Define

$$
\begin{aligned}
S_{r}(b ; w, I) & =\left(\frac{1}{|I|} \int_{I}\left|b-b_{I}\right|^{r} w^{r} d x\right)^{1 / r}, \\
\Lambda_{r}(f ; w, I) & =\left(\frac{1}{|I|} \int_{I}|f w|^{r}\right)^{1 / r}, \text { and } \\
K_{r}^{*}(b, f, w)(x) & =\sup _{1 \ni x} S_{r q^{\prime}}(b, w, I) \Lambda_{r q} l\left(f ; w^{-1}, I\right) .
\end{aligned}
$$

Also put $K^{*}=K_{1}^{*}$, and let $M_{\lambda}^{*}$ denote the weighted maximal function

$$
M_{\lambda}^{*} g(x)=\sup \left\{\frac{1}{\lambda(I)} \int_{I}|g| \lambda d y: x \in I\right\} .
$$

LeMma 4.4. For an appropriate choice of $q<p$, and for any $r$ with $1 \leqslant r<p / q$, there exists a weight $w$ depending on $r$ such that

(i) $w^{r q^{\prime}} \in\left(A_{q^{\prime}}\right)$, and

(ii) $\int\left[K_{r}^{*}(b, f, w)(x)\right]^{p} \lambda(x) d x \leqslant C \int|f|^{p} \mu(x) d x$. 
Proof. We will choose $w$ as $w=\mu^{1 / p} \lambda^{1 / p-1 / r q}$. To show (i), it will suffice to show that $w^{p^{\prime}} \in\left(A_{p^{\prime}}\right)$. For then (i) will hold by Reverse Hölder if $q$ is chosen sufficiently near $p$. For the $A_{p^{\prime}}$ condition, let $t>1$. Then

$$
\begin{aligned}
& \frac{1}{|I|} \int_{I} w^{p^{\prime}}\left(\frac{1}{|I|} \int_{I} w^{-p}\right)^{p^{\prime} / p}=\left(\frac{1}{|I|} \int_{I} \mu^{-p^{\prime} / p} \lambda^{p^{\prime} / p-p^{\prime} / r q}\right)\left(\frac{1}{|I|} \int_{I} \mu \lambda^{p / r q-1}\right)^{p^{\prime} / p} \\
& \leqslant {\left[\frac{1}{|I|} \int_{I} \mu^{-t p^{\prime} / p}\left(\frac{1}{|I|} \int_{I} \mu^{t}\right)^{p^{\prime} / p}\right]^{1 / t} } \\
& {\left[\frac{1}{|I|} \int_{I} \lambda^{t^{\prime}\left(p^{\prime} / p-p^{\prime} / r q\right)}\left(\frac{1}{|I|} \int_{I} \lambda^{t^{\prime}(p / r q-1)}\right)^{p^{\prime} / p^{\prime}}\right]^{1 / t^{\prime}} . }
\end{aligned}
$$

The first term

$$
\left[\frac{1}{|I|} \int_{I} \mu^{-t p^{\prime} / p}\left(\frac{1}{|I|} \int_{I} \mu^{t}\right)^{p^{\prime} / p}\right]^{1 / t}
$$

is bounded by Reverse Hölder and $\mu \in\left(A_{p}\right)$ for $t$ near one, say $t \leqslant t_{0}$. For the second term, consider the exponent $t^{\prime}(p / r q-1)$. As $q \rightarrow p, r \rightarrow 1$, and $p / r q \rightarrow 1$. So we can choose $q$ sufficiently near $p$ so that choosing $t^{\prime}$ with $t^{\prime}(p / r q-1)=1$ still keeps $t \leqslant t_{0}$ for $r=1$, and hence for $r>1$ as well. Then the second term is

$$
\left[\frac{1}{|I|} \int_{I} \bar{\lambda}^{p^{\prime} / p}\left(\frac{1}{|I|} \int_{I} \lambda\right)^{p^{\prime} / p}\right]^{1 / t^{\prime}},
$$

which is bounded, since $\lambda \in\left(A_{p}\right)$. Hence $w^{p^{\prime}} \in\left(A_{p^{\prime}}\right)$ and (i) follows.

We will show that

$$
\left(\frac{1}{|I|} \int_{I} \lambda\right)^{1 / r q} S_{r q^{\prime}}(b ; w, I) \leqslant C \text { for all } I .
$$

Assume (4) for the moment. For each $x$, there exist intervals $I_{x}$ containing $x$ which approximate $K_{r}^{*}$, that is

$$
\int\left[K_{r}^{*}(b, f, w)(x)\right]^{p} \lambda(x) d x \leqslant 2 \int\left[S_{r q^{\prime}}\left(b ; w, I_{x}\right) \Lambda_{r q}\left(f ; w^{-1}, I_{x}\right)\right]^{p} \lambda .
$$

Now

$$
\begin{aligned}
\Lambda_{r q}\left(f ; w^{-1}, I_{x}\right) & =\left(\frac{1}{|I|} \int_{I_{x}}|f|^{r q} w^{-r q} \lambda^{-1} \lambda\right)^{1 / r q} \\
& =\left(\lambda_{I_{*}}\right)^{1 / r q}\left(\frac{1}{\lambda\left(I_{x}\right)}\left|f w^{-1}\right|^{r q} \lambda^{-1} \lambda\right)^{1 / r q} \\
& \leqslant\left(\lambda_{I_{*}}\right)^{1 / r q}\left[M_{\lambda}^{*}\left(\left|f w^{-1}\right|^{r q} \lambda^{-1}\right)(x)\right]^{1 / r q},
\end{aligned}
$$


so that, by (4),

$$
\begin{aligned}
\int K_{r}^{*}(b, f, w)^{p} \lambda & \leqslant C \int\left[M_{\lambda}^{*}\left(\left|f w^{-1}\right|^{r q} \lambda^{-1}\right)\right]^{p / r q} \lambda \\
& \leqslant C \int\left|f w^{-1}\right|^{p} \lambda^{-p / r q} \lambda,
\end{aligned}
$$

by the boundedness of the Hardy-Littlewood maximal function,

$$
=C \int|f|^{p} \mu \text {. }
$$

So to show (ii), we must only verify (4). First,

$$
S_{r q^{\prime}}^{r q^{\prime}}(b ; w, I)=\frac{1}{|I|} \int_{I}\left|b-b_{I}\right|^{r q^{\prime}} \mu^{-r q^{\prime} / p} \lambda^{-q^{\prime}(1 / q-r / p)} .
$$

Choose $s$ so that $s q^{\prime}(1 / q-r / p)=p^{\prime} / p$. $q$ near $p$ means that $s$ is large, so that $r q^{\prime} s^{\prime} \leqslant p^{\prime}+\varepsilon$, the exponent in Lemma 4.3. Hence,

$$
\begin{aligned}
S_{r q^{\prime}}^{r q^{\prime}}(b ; w, I) & \leqslant\left(\frac{1}{|I|} \int_{I}\left|b-b_{I}\right|^{r q^{\prime} s^{\prime}} \mu^{r q^{\prime} s^{\prime} / p}\right)^{1 / s^{\prime}}\left(\frac{1}{|I|} \int_{I} \lambda^{-p^{\prime} / p}\right)^{1 / s} \\
& \leqslant C\left(\frac{1}{|I|} \int_{I} \lambda^{-1 / p}\right)^{r q^{\prime}}\left(\frac{1}{|I|} \int_{I} \lambda^{-p^{\prime} / p}\right)^{1 / s}, \text { by Lemma 4.3, } \\
& \leqslant C\left(\frac{1}{|I|} \int_{I} \lambda^{-p^{\prime} / p}\right)^{r q^{\prime} / p^{\prime}+1 / s}=C\left(\frac{1}{|I|} \int_{I} \lambda^{-p^{\prime} / p}\right)^{p q^{\prime} / p^{\prime} q}
\end{aligned}
$$

or

$$
S_{r q^{\prime}}(b ; w, I) \leqslant C\left[\left(\frac{1}{|I|} \int_{I} \lambda^{-p^{\prime} / p}\right)^{p / p^{\prime}}\right]^{1 / r q}
$$

and (4) holds by the $A_{p}$ condition.

The main ingredient of the proof is an estimate of the sharp function of $\left[H, M_{b}\right] f$, set out in the lemma below.

Lemma 4.5. Let $w$ and $\tilde{w}$ be weights with $w^{q^{\prime}}, \tilde{w}^{r q^{\prime}} \in\left(A_{q^{\prime}}\right)$ for some $r>1$. Then

$$
\begin{aligned}
\left(\left[H, M_{b}\right] f\right)^{\#}(x) \leqslant C[ & K^{*}(b, f, w)(x)+K^{*}(b, H f, w)(x) \\
& \left.+K_{r}^{*}(b, f, \tilde{w})(x)+\left(M_{\lambda}^{*}\left(|f \nu|^{q}\right)(x)\right)^{1 / q}\right] .
\end{aligned}
$$

Proof. Let $g=\left[H, M_{b}\right] f$. We must estimate $g^{\#}$. So fix $x$ and $I$ containing $x$. Let $x_{0}$ be the center of $I$. Define $f_{1}=f \chi_{2 I}$, and $f_{2}=f-f_{1}$. For any constant $c$,

$$
\frac{1}{|I|} \int_{I}\left|g-g_{I}\right| \leqslant \frac{2}{|I|} \int_{I}|g-c| \text {. }
$$

In particular,

$$
\frac{1}{|I|} \int_{I}\left|g-g_{I}\right| \leqslant \frac{2}{|I|} \int_{I}\left|g-H\left(b-b_{I}\right) f_{2}\left(x_{0}\right)\right| .
$$


Now

$$
\begin{aligned}
g & =\left[H, M_{b}\right] f=\left[H, M_{b-b_{I}}\right] f \\
& =H\left(b-b_{I}\right) f_{1}+H\left(b-b_{I}\right) f_{2}-\left(b-b_{I}\right) H f
\end{aligned}
$$

so that

$$
\begin{aligned}
\frac{1}{|I|} \int_{I}\left|g-g_{I}\right| \leqslant & \frac{2}{|I|} \int\left|b-b_{I}\right||H f|+\frac{2}{|I|} \int_{I}\left|H\left(b-b_{I}\right) f_{1}\right| \\
& +\frac{2}{|I|} \int_{I}\left|H\left(b-b_{I}\right) f_{2}(t)-H\left(b-b_{I}\right) f_{2}\left(x_{0}\right)\right| d t \\
= & 2\left(K_{1}+K_{2}+K_{3}\right) .
\end{aligned}
$$

For these terms, first

$$
\begin{aligned}
K_{1} & =\frac{1}{|I|} \int_{I}\left|b-b_{I}\right| w|H f| w^{-1} \\
& \leqslant\left(\frac{1}{|I|} \int_{I}\left|b-b_{I}\right|^{q^{\prime}} w^{q^{\prime}}\right)^{1 / q^{\prime}}\left(\frac{1}{|I|} \int_{I}|H f|^{q} w^{-q}\right)^{1 / q} \\
& =S_{q^{\prime}}(b ; w, I) \Lambda_{q}\left(H f ; w^{-1}, I\right) \leqslant K^{*}(b, H f, w)(x) .
\end{aligned}
$$

For the second piece,

$$
\begin{aligned}
K_{2} & \leqslant\left(\frac{1}{|I|} \int_{I}\left|H\left(b-b_{I}\right) f_{1}\right|^{r}\right)^{1 / r} \\
& \leqslant|I|^{-1 / r}\left(\int_{0}^{2 \pi}\left|H\left(b-b_{I}\right) f_{1}\right|\right)^{1 / r} \\
& \leqslant C|I|^{-1 / r}\left(\int\left|b-b_{I}\right|^{r}\left|f_{1}\right|^{r}\right)^{1 / r}, \text { by the Theorem of M. Riesz, } \\
& =2^{1 / r} C\left(\frac{1}{|2 I|} \int_{2 I}\left|b-b_{I}\right|^{r}|f|^{r}\right)^{1 / r} \\
& \leqslant 2^{1 / r} C\left[\left(\frac{1}{|2 I|} \int_{2 I}\left|b-b_{2 I}\right|^{r}|f|^{r}\right)^{1 / r}+\left|b_{I}-b_{2 I}\right|\left(\frac{1}{|2 I|} \int_{2 I}|f|^{r}\right)^{1 / r}\right] \\
& =2^{1 / r} C[A+B] .
\end{aligned}
$$

Here,

$$
\begin{aligned}
A & =\left(\frac{1}{|2 I|} \int_{2 I}\left|b-b_{I}\right|^{r} \tilde{w}^{r}|f|^{r} \tilde{w}^{-r}\right)^{1 / r} \\
& \leqslant S_{r q^{\prime}}(b ; \tilde{w}, 2 I) \Lambda_{r q}\left(f ; \tilde{w}^{-1}, 2 I\right) \leqslant K_{r}^{*}(b, f, \tilde{w})(x) .
\end{aligned}
$$


To estimate $B$,

$$
\begin{aligned}
\left|b_{I}-b_{2 I}\right| & \leqslant \frac{1}{|I|} \int_{I}\left|b-b_{2 I}\right| \leqslant \frac{2}{|2 I|} \int_{2 I}\left|b-b_{2 I}\right| \\
& \leqslant 2 S_{q^{\prime}}(b ; \tilde{w}, 2 I)\left(\frac{1}{|2 I|} \int_{2 I} \tilde{w}^{-q}\right)^{1 / q} \\
& \leqslant 2 S_{r q^{\prime}}(b ; \tilde{w}, 2 I)\left(\frac{1}{|2 I|} \int_{2 I} \tilde{w}^{-r q}\right)^{1 / r q}
\end{aligned}
$$

so that

$$
\begin{aligned}
B & \leqslant 2 S_{r q^{\prime}}(b ; \tilde{w}, 2 I)\left(\frac{1}{|2 I|} \int_{2 I} \tilde{w}^{-r q}\right)^{1 / r q}\left(\frac{1}{|2 I|} \int_{2 I}|f|^{r} \tilde{w}^{-r} \tilde{w}^{r}\right)^{1 / r} \\
& \leqslant 2 S_{r q^{\prime}}(b ; \tilde{w}, 2 I) \Lambda_{r q}\left(f ; \tilde{w}^{-1}, 2 I\right)\left(\frac{1}{|2 I|} \int_{2 I} \tilde{w}^{-r q}\right)^{1 / r q}\left(\frac{1}{|2 I|} \int_{2 I} \tilde{w}^{r q^{\prime}}\right)^{1 / r q^{\prime}} \\
& \leqslant C K_{r}^{*}(b, f, \tilde{w})(x)
\end{aligned}
$$

since $w^{r q^{\prime}} \in\left(A_{q^{\prime}}\right)$. Hence $K_{2} \leqslant C K_{r}^{*}(b, f, \tilde{w})(x)$. For $K_{3}$, let $t \in I$. Then

$$
\begin{aligned}
& \left|H\left(b-b_{I}\right) f_{2}(t)-H\left(b-b_{I}\right) f_{2}\left(x_{0}\right)\right| \\
& \quad \leqslant \int\left|Q(t-y)-Q\left(x_{0}-y\right)\right|\left|b-b_{I}\right|\left|f_{2}\right| d y \\
& \quad \leqslant C \int \frac{\left|t-x_{0}\right|}{|t-y|\left|x_{0}-y\right|}\left|f_{2}(y)\right|\left|b-b_{I}\right| d y \\
& \quad=C \int_{\left|x_{0}-y\right|>\delta} \frac{t-x_{0}}{|t-y|\left|x_{0}-y\right|}|f|\left|b-b_{I}\right|
\end{aligned}
$$

where $\delta=|I|$.

Since $t \in I$,

$$
\left|t-x_{0}\right| \leqslant \delta / 2 \leqslant \frac{1}{2}\left|x_{0}-y\right|
$$

and

$$
|t-y| \geqslant\left|x_{0}-y\right|-\left|t-x_{0}\right| \geqslant \frac{1}{2}\left|x_{0}-y\right|
$$

Hence,

$$
\left|H\left(b-b_{I}\right) f_{2}(t)-H\left(b-b_{I}\right) f_{2}\left(x_{0}\right)\right| \leqslant C \delta \int_{\left|x_{0}-y\right|>\delta} \frac{1}{\left|x_{0}-y\right|^{2}}|f|\left|b-b_{I}\right| d y,
$$

and since this holds for all $t \in I$, the same bound must hold for $K_{3}$. Therefore,

$$
\begin{aligned}
K_{3} & \leqslant C \delta \sum_{k} \int_{2^{k-1} \delta<\left|x_{0}-y\right| \leqslant 2^{k} \delta} \frac{1}{\left|x_{0}-y\right|^{2}}|f|\left|b-b_{I}\right| d y \\
& \leqslant C \sum_{k} 2^{2-2 k} \delta^{-1} \int_{\left|x_{0}-y\right| \leqslant 2^{k} \delta}|f|\left|b-b_{I}\right| d y .
\end{aligned}
$$


Let $I_{k}=2^{k} I$. Then

$$
\begin{aligned}
K_{3} & \leqslant 8 C \sum_{k} 2^{-k} \frac{1}{\left|I_{k+1}\right|} \int_{I_{k+1}}\left|b-b_{I}\right||f| \\
& \leqslant 16 C \sum_{k} 2^{-k}\left(\frac{1}{\left|I_{k}\right|} \int_{I_{k}}\left|b-b_{I_{k}}\right||f|+\frac{1}{\left|I_{k}\right|} \int_{I_{k}}\left|b_{I}-b_{I_{k}}\right||f|\right) \\
& =16 C \sum_{k} 2^{-k}\left(L_{k}+M_{k}\right) .
\end{aligned}
$$

But

$$
L_{k} \leqslant S_{q^{\prime}}\left(b ; w, I_{k}\right) \Lambda_{q}\left(f ; w^{-1}, I_{k}\right) \leqslant K^{*}(b, f, w)(x),
$$

so that

$$
K_{3} \leqslant 16 C\left[K^{*}(b, f, w)(x)+\sum_{k} 2^{-k} M_{k}\right] .
$$

We must show

$$
\sum_{k} 2^{-k} M_{k} \leqslant C\left[M_{\lambda}^{*}\left(|f \nu|^{q}\right)(x)\right]^{1 / q} .
$$

To prove (5), we will use two facts. First, since $b \in \mathrm{BMO}_{\nu}$,

$$
\int_{J}\left|b-b_{J}\right| \leqslant C \nu(J) \text { for each interval } J,
$$

and second, since $\nu \in\left(A_{\infty}\right)$, there exists a $\delta>0$ such that

$$
\frac{\nu(E)}{\nu(J)} \leqslant C\left(\frac{|E|}{|J|}\right)^{\delta} \quad \text { for all measurable sets } E \subseteq J .
$$

Thus,

$$
\begin{aligned}
\left|b_{I}-b_{I_{k}}\right| & \leqslant \sum_{n=0}^{k-1}\left|b_{I_{n}}-b_{I_{n+1}}\right| \leqslant \sum_{n=0}^{k-1} \frac{1}{\left|I_{n}\right|} \int_{I_{n}}\left|b-b_{I_{n+1}}\right| \\
& \leqslant 2 \sum_{n=0}^{k-1} \frac{1}{\left|I_{n+1}\right|} \int_{I_{n+1}}\left|b-b_{I_{n}, 1}\right| \\
& \leqslant C \sum_{n=0}^{k-1} \frac{\nu\left(I_{n+1}\right)}{\left|I_{n+1}\right|} \text { by }(2) \\
& \leqslant C \nu_{I_{k}} \sum_{n=0}^{k-1} \frac{\nu\left(I_{n+1}\right)}{\nu\left(I_{k}\right)} \cdot \frac{\left|I_{k}\right|}{\left|I_{n+1}\right|} \\
& \leqslant C \nu_{I_{k}} \sum_{n=0}^{k-1}\left(\frac{\left|I_{k}\right|}{\left|I_{n+1}\right|}\right)^{1-\delta} \text { by }(3), \\
& =C \nu_{I_{k}} \sum_{n=0}^{k-1} 2^{(k-n-1)(1-\delta)} \leqslant C \nu_{I_{k}} 2^{k(1-\delta)},
\end{aligned}
$$


and hence

$$
\begin{aligned}
\sum_{k} 2^{-k} M_{k} & \leqslant C \sum_{k} 2^{-k \delta} \nu_{I_{k}} \frac{1}{\left|I_{k}\right|} \int_{I_{k}}|f| \\
& \leqslant C \sum_{k} 2^{-k \delta} \nu_{I_{k}}\left(\frac{1}{\left|I_{k}\right|} \int_{I_{k}}|f \nu|^{q} \lambda\right)^{1 / q}\left(\frac{1}{\left|I_{k}\right|} \int_{I_{k}} \nu^{-q^{\prime}} \lambda^{-q^{\prime} / q}\right)^{1 / q^{\prime}} \\
& =C \sum_{k} 2^{-k \delta} \nu_{I_{k}}\left(\lambda_{I_{k}}\right)^{1 / q}\left(\frac{1}{\lambda\left(I_{k}\right)} \int_{I_{k}}|f \nu|^{q} \lambda\right)^{1 / q}\left(\frac{1}{\left|I_{k}\right|} \int_{I_{k}} \nu^{-q^{\prime}} \lambda^{-q^{\prime} / q}\right)^{1 / q^{\prime}} \\
& \leqslant C\left[M_{\lambda}^{*}\left(|f \nu|^{q}\right)(x)\right]^{1 / q} \sum_{k} 2^{-k \delta} \nu_{I_{k}}\left(\lambda_{I_{k}}\right)^{1 / q}\left(\frac{1}{\left|I_{k}\right|} \int_{I_{k}} \nu^{-q^{\prime}} \lambda^{-q^{\prime} / q}\right)^{1 / q^{\prime}} .
\end{aligned}
$$

So (5) will hold provided

$$
\nu_{I}\left(\lambda_{I}\right)^{1 / q}\left(\frac{1}{|I|} \int_{I} \nu^{-q^{\prime}} \lambda^{-q^{\prime} / q}\right)^{1 / q^{\prime}} \leqslant C \text { for all } I .
$$

To show (8),

$$
\nu^{-q^{\prime}} \lambda^{-q^{\prime} / q}=\mu^{-q^{\prime} / p} \lambda^{-q^{\prime}(1 / q-1 / p)} .
$$

Choose $s$ so that $s q^{\prime}(1 / q-1 / p)=p^{\prime} / p$. So $s$ is large, and Reverse Hölder will apply to $\mu^{-p^{\prime} / p}$ with exponent $q^{\prime} s^{\prime} / p^{\prime}$ for $q$ near $p$. So

$$
\begin{aligned}
& \leqslant\left(\lambda_{I}\right)^{1 / q}\left(\mu_{I}\right)^{1 / p}\left(\lambda_{I}^{-p^{\prime} / p}\right)^{1 / p^{\prime}}\left(\frac{1}{|I|} \int_{I} \mu \frac{-q^{\prime} s^{\prime}}{p}\right)^{1 / s^{\prime} q^{\prime}}\left(\frac{1}{|I|} \int_{I} \lambda^{-p^{\prime} / p}\right)^{1 / s q^{\prime}} \\
& \leqslant C\left(\lambda_{I}\right)^{1 / q}\left(\mu_{I}\right)^{1 / p}\left(\lambda_{I}^{-p^{\prime} / p}\right)^{1 / p^{\prime}+1 / s q^{\prime}}\left(\mu^{-p^{\prime} / p}\right)^{1 / p^{\prime}}, \quad \text { by Reverse Hölder, } \\
& =C\left(\lambda_{I}\right)^{1 / q}\left(\lambda_{I}^{-p^{\prime} / p}\right)^{p / p^{\prime} q}\left(\mu_{I}\right)^{1 / p}\left(\mu_{I}^{-p^{\prime} / p}\right)^{1 / p^{\prime}}
\end{aligned}
$$

which is bounded, since $\mu$ and $\lambda$ are in $\left(A_{p}\right)$.

Proof of Theorem 4.2, PART (i). By Lemma 4.5,

$$
\begin{aligned}
\int\left(\left[H, M_{b}\right] f\right)^{\# p} \lambda \leqslant C\left[\int\right. & K^{*}(b, f, w)^{p} \lambda+\int K^{*}(b, H f, w)^{p} \lambda \\
& \left.+\int K_{r}^{*}(b, f, \tilde{w})^{p} \lambda+\int\left(M_{\lambda}^{*}\left(|f \nu|^{q}\right)\right)^{p / q} \lambda\right],
\end{aligned}
$$

for $w$ and $\tilde{w}$ satisfying $w^{q^{\prime}}$ and $\tilde{w}^{r q^{\prime}} \in\left(A_{q^{\prime}}\right)$. By Lemma 4.4, we can choose an $r>1$ and such weights $w$ and $\tilde{w}$ so that

$$
\int K^{*}(b, f, w)^{p} \lambda \leqslant C \int|f|^{p} \mu
$$

and

$$
\int K_{r}^{*}(b, f, \tilde{w})^{p} \lambda \leqslant \int|f|^{p} \mu .
$$


Therefore,

$$
\begin{aligned}
& \int\left[\left(\left[H, M_{b}\right] f\right)^{\#}(x)\right]^{p} \lambda(x) \\
& \quad \leqslant C\left[\int|f|^{p} \mu+\int|H f|^{p} \mu+\int M_{\lambda}\left(|f \mu|^{q}\right)^{p / q} \lambda\right] .
\end{aligned}
$$

By the Theorem of Hunt, Muckenhoupt, and Wheeden,

$$
\int|H f|^{p} \mu \leqslant C \int|f|^{p} \mu
$$

and by Hardy and Littlewood's Theorem,

$$
\int M_{\lambda}^{*}\left(|f \nu|^{q}\right)^{p / q} \lambda \leqslant C \int|f \nu|^{p} \lambda=C \int|f|^{p} \mu .
$$

So we conclude

$$
\int\left(\left[H, M_{b}\right] f\right)^{\# p} \lambda \leqslant C \int|f|^{p} \mu .
$$

Now let

$$
k=\frac{1}{2 \pi} \int_{0}^{2 \pi}\left[H, M_{b}\right] f .
$$

By the Sharp Function Theorem,

$$
\int\left|\left[H, M_{b}\right] f-k\right| \lambda \leqslant C \int|f|^{p} \mu .
$$

Thus,

$$
\begin{aligned}
\left(\int\left|\left[H, M_{b}\right] f\right|^{p} \lambda\right)^{1 / p} & \leqslant\left(\int\left|\left[H, M_{b}\right] f-k\right|^{p} \lambda\right)^{1 / p}+k\left(\int \lambda\right)^{1 / p} \\
& \leqslant C\left(\int|f|^{p} \mu\right)^{1 / p}+k\left(\int \lambda\right)^{1 / p} .
\end{aligned}
$$

Finally, $|k|=(1 / 2 \pi) \int_{T}\left[H, M_{b-b_{T}}\right] f \mid$, and estimating this just as in Lemma 4.5 for $K_{1}$ and $K_{2}$ gives

$$
\begin{aligned}
|k| & \leqslant \frac{I}{2 \pi} \int_{T}\left|b-b_{T}\right||H f|+\frac{1}{2 \pi} \int_{T}\left|H\left(b-b_{T}\right) f\right| \\
& \leqslant\left[K^{*}(b, H f, \omega)(x)+K^{*}(b, f, \tilde{\omega})(x)\right]
\end{aligned}
$$

for any $x \in T$, so that

$$
\begin{aligned}
\left(\int_{T}|k|^{\rho} \lambda\right)^{1 / \rho} & \leqslant\left[\left(\int_{T} K^{*}(b, H f, \omega)^{p} \lambda\right)^{1 / \rho}+\left(\int_{T} K^{*}(b, f, \tilde{\omega})^{\rho} \lambda\right)^{1 / \rho}\right] \\
& \leqslant\left(\|H f\|_{L^{\rho}(\mu)}+\|f\|_{L^{\rho}(\mu)}\right) \leqslant\|f\|_{L^{\rho}(\mu)}
\end{aligned}
$$

by Lemma 4.4 with appropriate choices of $\omega, \tilde{\omega}$ and $r>1$, and by the Hunt, Muckenhoupt, and Wheeden Theorem, and part (i) follows. 
Proof of Theorem 4.2, PART (ii). We may assume that

$$
\int\left|\left[H, M_{b}\right] f\right|^{p} \lambda \leqslant \int|f|^{p} \mu
$$

and that $b$ is real. Fix $I$, centered at $x_{0}$. Since $\left[H, M_{b}\right]=\left[H, M_{b-b_{l}}\right]$, we may further assume that $b_{I}=0$. Put $M=(1 / \nu(I)) \int_{I}|b|$. We must bound $M$, independent of $I$. So we can assume that $M$ is quite large. Let $E=\{x \in I: b(x) \geqslant 0\}$. We may assume without loss of generality that $|E| \geqslant \frac{1}{2}|I|$. Let $E^{\prime} \subseteq E$ have measure $\left|E^{\prime}\right|=|I \sim E|$. Define $\psi$ by $\psi=\chi_{E^{\prime}}-\chi_{I \sim E}$. Then $\psi b \geqslant 0$, and $\int \psi=0$. Also, since $\int_{I} b=0$, $\int_{I \sim E}(-b)=\int_{E} b$. Hence,

$$
\int \psi b \geqslant \int_{I \sim E}(-b)=\frac{1}{2}\left(\int_{i \sim E}(-b)+\int_{E} b\right)=\frac{1}{2} \int_{I}|b|=\frac{1}{2} M \nu(I) .
$$

Now let $x \in 2 I$. Then

$$
\left|\left[H, M_{b}\right] \psi(x)\right| \geqslant|H(b \psi)(x)|-|b(x)||H \psi(x)| .
$$

To analyze this,

$$
\begin{aligned}
|H(b \psi)(x)| & =\int_{I}|Q(x-y)|(b \psi)(y) d y \\
& \geqslant \frac{2 C_{1}}{\left|x-x_{0}\right|} \int_{I}(b \psi) \geqslant \frac{C_{1}}{\left|x-x_{0}\right|} M \nu(I),
\end{aligned}
$$

while

$$
\begin{aligned}
|H \psi(x)| & =\left|\int Q(x-y) \psi(y) d y\right| \\
& =\mid \int\left[Q(x-y)-Q\left(x-x_{0}\right] \psi(y) d y \mid, \quad \text { since } \int \psi=0,\right. \\
& \leqslant C_{2} \int_{I} \frac{\left|y-x_{0}\right|}{|x-y|\left|x-x_{0}\right|} d y \leqslant C_{2}|I|^{2}\left|x-x_{0}\right|^{-2},
\end{aligned}
$$

and we conclude,

$$
\left|\left[H, M_{b}\right] \psi(x)\right| \geqslant \frac{C_{1} M \nu(I)}{\left|x-x_{0}\right|}-\frac{C_{2}|I|^{2}|b(x)|}{\left|x-x_{0}\right|^{2}}, \quad x \in 2 I .
$$

Choose $\alpha<C_{1}$ and $\beta$ a small universal constant. We can think of the unit circle as $\left(x_{0}-\pi, x_{0}+\pi\right)$. $\beta$ will be chosen so small that $2 \beta M^{1 / p}|I|<\pi$. For otherwise,

$$
\frac{\pi}{2 \beta M^{1 / p}} \leqslant|I|=\int_{I} \nu^{1 / p} \nu^{-1 / p} \leqslant \nu(I)^{1 / p}\left(\int_{0}^{2 \pi} \nu^{-q / p}\right)^{1 / q},
$$

or

$$
\nu(I) \geqslant\left(\frac{\pi}{2 \beta M^{1 / p}}\right)^{p}\left(\int \nu^{-q / p}\right)^{-p / q}=\frac{1}{C \beta^{p} M},
$$

and so

$$
M=\frac{1}{\nu(I)} \int_{I}|b| \leqslant C \beta^{p} M\|b\|_{1} .
$$


We can choose $\beta$ sufficiently small so that $C \beta^{p}\|b\|_{1}<1$, leading to a contradiction. Now put

$$
\begin{aligned}
& J=\left\{x:|I|<\left(x-x_{0}\right)<\beta M^{1 / p}|I|\right\}, \\
& F=\left\{x \in J: C_{2}|b(x)||I|^{2}<\left(c_{1}-\alpha\right) M \nu(I)\left(x-x_{0}\right)\right\}, \text { and } \\
& G=J \sim F .
\end{aligned}
$$

By the argument above, $J \subseteq\left(x_{0}, x_{0}+\pi / 2\right)$, and so $2 J \subseteq T$, the unit circle. We can assume that $M$ is large enough so that $I \subseteq 2 J$. Then

$$
\begin{aligned}
\mu(I) & \geqslant \int|\psi|^{p} \mu \geqslant \int\left|\left[H, M_{b}\right] \psi\right|^{p} \lambda \geqslant \int_{F}\left|\left[H, M_{b}\right] \psi\right|^{p} \lambda \\
& \geqslant \int_{F}\left|\frac{c_{1} M \nu(I)}{x-x_{0}}-\frac{c_{2}|I|^{2} b(x) \mid}{\left(x-x_{0}\right)^{2}}\right|^{p} \lambda, \quad \text { by }(1), \\
& \geqslant \int_{F}\left[\frac{\alpha M \nu(I)}{x-x_{0}}\right]^{p} \lambda \geqslant \int_{F}\left[\frac{\alpha M \nu(I)}{\beta M^{1 / p}|I|}\right]^{p} \lambda \\
& =\lambda(F)\left(\frac{\alpha}{\beta} \nu_{I}\right)^{p} M^{p-1} \geqslant \lambda(F)\left(\frac{\alpha}{\beta} \nu_{I}\right)^{p},
\end{aligned}
$$

so that

$$
\begin{aligned}
\lambda(F) & \leqslant\left(\frac{\beta}{\alpha}\right)^{p}\left(\nu_{I}\right)^{-p} \mu(I) \leqslant\left(\frac{\beta}{\alpha}\right)^{p}\left(\frac{1}{|I|} \int_{I} \nu^{-1}\right)^{p} \mu(I) \\
& =\left(\frac{\beta}{\alpha}\right)^{p}\left(\frac{1}{|I|} \int_{I} \lambda^{1 / p} \mu^{-1 / p}\right)^{p} \mu(I) \\
& \leqslant\left(\frac{\beta}{\alpha}\right)^{p} \lambda_{I}\left(\frac{1}{|I|} \int_{I} \mu^{-q / p} \mu(I)\right), \quad \text { where } \frac{1}{p}+\frac{1}{q}=1, \\
& =\left(\frac{\beta}{\alpha}\right)^{p} \lambda(I) \mu_{I}\left(\mu^{-q / p}\right)_{I}^{p / q} \leqslant C\left(\frac{\beta}{\alpha}\right)^{p} \lambda(I), \text { by the } A_{p} \text { condition, } \\
& \leqslant C(\beta / \alpha)^{p} \lambda(2 J) .
\end{aligned}
$$

Now

$$
\begin{aligned}
\frac{|F|}{|2 J|} & =\frac{1}{|2 J|} \int_{F} \lambda^{1 / p} \lambda^{-1 / p} \leqslant\left(\frac{1}{|2 J|} \int_{F} \lambda\right)^{1 / p}\left(\frac{1}{|2 J|} \int_{2 J} \lambda^{-q / p}\right)^{1 / q} \\
& =\left[\frac{\lambda(F)}{\lambda(J)}\right]^{1 / p}\left(\frac{1}{|2 J|} \int_{2 J} \lambda\right)^{1 / p}\left(\frac{1}{|2 J|} \int_{2 J} \lambda^{-q / p}\right)^{1 / q} \\
& \leqslant C\left[\frac{\lambda(F)}{\lambda(2 J)}\right]^{1 / p}, \quad \text { by }\left(A_{p}\right), \\
& \leqslant C \frac{\beta}{\alpha}
\end{aligned}
$$


and thus $|F| \leqslant 2 C \beta / \alpha \cdot \beta M^{1 / p}|I|$. We will also require $\beta$ to be so small that $2 C \beta / \alpha \leqslant \frac{1}{3}$. Then for $M$ large,

$$
|G| \geqslant \frac{1}{2} \beta M^{1 / p}|I|
$$

Notice that $\beta$ does not depend on $M$ or $|I|$. Next let

$$
H^{*} f(x)=\int Q(x-y) f(y) d y .
$$

Then the adjoint of the commutator is $\left[H, M_{b}\right]^{*}=-\left[H^{*}, M_{b}\right]$. Also, if $T$ is any operator satisfying $\int|T f|^{p} \lambda \leqslant \int|f|^{p} \mu$, then any easy argument shows that its adjoint satisfies

$$
\left(\left|T^{*} f\right|^{q} \mu^{-q / p}\right) \leqslant\left(\int|f|^{q} \lambda^{-q / p}\right) .
$$

In particular, for $T=\left[H, M_{b}\right]$, we have

$$
\int\left|\left[H^{*}, M_{b}\right] g\right|^{q} \mu^{-q / p} \leqslant \int|g|^{q} \lambda^{-q / p} .
$$

Now let $g=(\operatorname{sgn} b) \chi_{G}$. Then for $x \in I$,

$$
\begin{aligned}
\left|\left[H^{*}, M_{b}\right] g(x)\right| & \geqslant \int_{G} Q(y-x)(b g)(y) d y-|b(x)|\left|H^{*} g(x)\right| \\
& \geqslant 2 c_{1} \int \frac{|b(y)|}{\left|y-x_{0}\right|}-|b(x)| \int_{G} Q(y-x) d y \\
& \geqslant \frac{2 c_{1}}{c_{2}}\left(c_{1}-\alpha\right) \frac{M \nu(I)}{|I|^{2}}|G|-c_{3}|b(x)| \int_{1} \beta M^{1 / p}|I| \frac{d y}{y} \\
& \geqslant C M^{1+1 / p_{\nu}}-C^{\prime}|b(x)| \log M, \quad \text { by }(2) .
\end{aligned}
$$

Let $D=\left\{x \in I:|b(x)| \leqslant 2 M \nu_{I}\right\}$. Then for $x \in D$,

$$
\begin{aligned}
\left|\left[H^{*}, M_{b}\right] g(x)\right| & \geqslant \nu_{I}\left[C M^{1+1 / p}-2 C^{\prime} M \log M\right] \\
& \geqslant C \nu_{I} M^{1+1 / p} \quad \text { for large } M .
\end{aligned}
$$

Next,

$$
2 M|I \sim D|=\int_{I \sim D} 2 M \leqslant|I| \int_{I} \frac{|b|}{\nu(I)} \leqslant M|I|,
$$

so that $|I \sim D| \leqslant \frac{1}{2}|I|$, or $|D| \geqslant \frac{1}{2}|I|$. By (11),

$$
\int\left|\left[H^{*}, M_{b}\right] g\right|^{q} \mu^{-q / p} \leqslant \int|g|^{q} \lambda^{-q / p} \leqslant \int_{G} \lambda^{-q / p} \leqslant \int_{2 J} \lambda^{-q / p} .
$$

Thus,

$$
\int_{2 J} \lambda^{-q / p} \geqslant \int_{D}\left|\left[H^{*}, M_{b}\right] g\right|^{q} \mu^{-q / p} \geqslant C\left(\nu_{I}\right)^{q} M^{q+q / p} \int_{D} \mu^{-q / p}
$$

But

$$
\frac{1}{2} \leqslant \frac{|D|}{|I|}=\frac{1}{|I|} \int_{D} \mu^{1 / p} \mu^{-1 / p} \leqslant\left(\mu_{I}\right)^{1 / p}\left(\frac{1}{|I|} \int_{D} \mu^{-q / p}\right)^{1 / q}
$$


so that $(1 /|I|) \int_{D} \mu^{-q / p} \geqslant 2^{-q}\left(\mu_{I}\right)^{-q / p}$, and thus

$$
\frac{1}{|I|} \int_{2 J} \lambda^{-q / p} \geqslant C\left(\nu_{1}\right)^{q} M^{q+q / p} 2^{-q}\left(\mu_{I}\right)^{-q / p},
$$

or

$$
\begin{aligned}
M^{q+q / p} & \leqslant C \frac{1}{|I|} \int_{2 J} \lambda^{-q / p}\left(\mu_{I}\right)^{q / p}\left(\nu_{I}\right)^{-q} \\
& \leqslant C \frac{1}{|I|} \int_{2 J} \lambda^{-q / p}\left(\mu_{I}\right)^{q / p}\left(\frac{1}{|I|} \int_{I} \nu^{-1}\right)^{q} \\
& =C \frac{|2 J|}{|I|}\left(\lambda^{-q / p}\right)_{2 J}\left(\mu_{I}\right)^{q / p}\left(\frac{1}{|I|} \int_{I} \lambda^{1 / p} \mu^{-1 / p}\right)^{q} \\
& \leqslant C \frac{|2 J|}{|I|}\left(\lambda^{-q / p}\right)_{2 J}\left(\mu_{I}\right)^{q / p}\left(\mu^{-q / p}\right)_{I}\left(\frac{1}{|I|} \int_{I} \lambda\right)^{q / p} \\
& \leqslant C\left(\frac{|2 J|}{|I|}\right)^{1+q / p}\left(\mu_{I}\right)^{q / p}\left(\mu^{-q / p}\right)_{I}\left(\lambda_{2 J}\right)^{q / p}\left(\lambda^{-q / p}\right)_{2 J} \\
& \leqslant C\left(\frac{|2 J|}{|I|}\right)^{1+q / p}, \quad \text { as } \lambda, \mu \in\left(A_{p}\right), \\
& \leqslant C\left(2 \beta M^{1 / p}\right)^{1+q / p} .
\end{aligned}
$$

So we have

$$
C \geqslant M^{q+(q / p)(1-1 / p)-1 / p}=M^{q}
$$

and we have an upper bound on $M$.

V. A weighted norm inequality for vectors. Let $W$ be a symmetric, positive definite, $n \times n$ matrix-valued function on the unit circle $T . W(x)$ induces a pointwise inner product on the vector space $C^{n}$ given by $(f, g)_{W(x)}=(W(x) f, g)$ where the latter is the standard dot product on $C^{n}$. This extends to vector-valued functions as

$$
(f, g)_{W}=\frac{1}{2 \pi} \int_{T}(W(x) f(x), g(x)) d x .
$$

This inner product in turn induces a Hilbert space $L^{2}(W)$ of vector-valued functions whose $W$-norm is finite.

We wish to extend Theorem 1.2 to this setting. For what weights $W$ is the conjugate operator $H$ a bounded operator on $L^{2}(W)$ ? Nonconstructive necessary and sufficient conditions have been found by Pousson [9] and Rabindranathan [10] using the Hilbert space arguments of Helson and Szegö [4]. We will present a sufficient condition which is constructive, and which can be generalized to appropriately defined $L^{p}(W)$ spaces [1].

THEOREM 5.1. Let $W=U^{*} \Lambda U$, where $U$ is unitary, $\Lambda$ diagonal, and the diagonal entries $\lambda_{k}$ of $\Lambda$ are $A_{2}$ weights. If for each $r$ and $j$,

$$
u_{r j} \in \mathrm{BMO}_{\left(\lambda_{r} \lambda_{k}^{-1}\right)^{1 / 2}} \text { for } k=1,2, \ldots, n,
$$

then $H$ is a bounded operator on $L^{2}(W)$. 
Proof. The $W$-norm of $H f$ is given by

$$
\|H f\|=\sum_{k} \frac{1}{2 \pi} \int_{T}\left|(U H f)_{k}(x)\right|^{2} \lambda_{k}(x) d x .
$$

We will bound each $\left|(U H f)_{k}\right|$.

$$
\begin{aligned}
U H f & =H(U f)+U H f-H(U f) \\
& =H(U f)+U H U^{*}(U f)-U U^{*} H U f,
\end{aligned}
$$

so that, with $U=\left(u_{r j}\right)$,

$$
(U H f)_{k}=H(U f)_{k}+\sum_{j} u_{k j}\left(H U^{*}(U f)_{j}-U^{*} H(U f)_{j}\right) .
$$

Now

$$
\begin{aligned}
H U^{*}(U f)_{j}-U^{*} H(U f)_{j} & =\sum_{r} \bar{u}_{r j}(U f)_{r}-\bar{u}_{r j} H(U f)_{r} \\
& =\sum_{r}\left[H, M_{\bar{u}_{r j}}\right](U f)_{r} .
\end{aligned}
$$

Since $U$ is unitary, each $\left|u_{k j}\right| \leqslant 1$. Thus

$$
\left|(U H f)_{k}\right| \leqslant\left|H(U f)_{k}\right|+\sum_{r, j}\left|\left[H, M_{\bar{u}_{r j}}\right](U f)_{r}\right|,
$$

and so

$$
\|H f\|_{W}^{2} \leqslant C\left(\sum_{k} \int\left|H(U f)_{k}\right|^{2} \lambda_{k}+\sum_{r, j, k} \int \|\left.\left[H, M_{\bar{u}_{r j}}\right](U f)_{r}\right|^{2} \lambda_{k}\right)
$$

where $c$ depends only on the dimension $n$.

By the Hunt, Muckenhoupt and Wheeden Theorem 1.2,

$$
\int\left|H(U f)_{k}\right|^{2} \lambda_{k} \leqslant C \int\left|(U f)_{k}\right|^{2} \lambda_{k}
$$

and by the Commutator Theorem 4.2,

$$
\int\left|\left[H, M_{\tilde{u}_{r j}}\right](U f)_{r}\right|^{2} \lambda_{k} \leqslant C \int\left|(U f)_{r}\right|^{2} \lambda_{r},
$$

since each $\bar{u}_{r j} \in \mathrm{BMO}_{\left(\lambda, \lambda_{k}^{-1}\right)^{1 / 2}}$ by assumption. So

$$
\|H f\|_{w}^{2} \leqslant C \sum_{r} \int\left|(U f)_{r}\right|^{2} \lambda_{r}=C\|f\|_{w}^{2} .
$$

We close with some remarks on the converse of Theorem 5.1. The requirement that the $\lambda_{k}$ be $A_{2}$ weights causes no great pain. There are examples of good weights with a diagonalization for which the diagonal entries are not in $A_{2}$, but these examples reflect a choice in diagonalization rather than the structure of the weight. In particular, if $U^{*} \Lambda U$ is a good weight with $U$ continuous, the $\lambda_{k}$ 's must be in $A_{2}$ [1].

In any converse to Theorem 5.1, very little can be said about the arguments of the unitary entries. For if $U^{*} \Lambda U$ is a good weight, and if $J$ is any diagonal, unitary matrix, then $U^{*} J^{*} \Lambda J U=U^{*} \Lambda U$, so that necessary conditions must apply to $J U$ as well as $U$. Multiplication by $J$ smears the arguments of each row. 
The condition that we suspect is necessary is the following

Conjecture 5.2 Let $H$ be a bounded operator on $L^{2}\left(U^{*} \Lambda U\right)$, where

$$
\Lambda=\left[\begin{array}{lll}
\lambda_{1} & & 0 \\
& \ddots & \\
0 & & \lambda_{n}
\end{array}\right]
$$

is diagonal, and $U=\left(u_{i j}\right)$ is unitary. Then each $\left|u_{r j}\right| \in \mathrm{BMO}_{\left(\lambda_{r} \lambda_{k}^{-1}\right)^{1 / 2}}, k=1,2, \ldots, n$.

This author has also studied the simpler moving average operator

$$
A_{h} f(x)=\frac{1}{2 h} \int_{x-h}^{x+h} f(t) d t .
$$

A weight $W$ is said to be a good weight for the moving average if $A_{h}$ is bounded on $L^{2}(W)$, with bound independent of $h$.

Conjecture 5.2 holds for the moving average in two dimensions, but our proof breaks down in higher dimensions [1].

Similarly, one can ask these questions about the Hardy-Littlewood maximal function, defined in the vector setting to maximize the $W$-norm.

One example motivated much of these ideas. Let $\alpha, \beta>0, \alpha<1$. Put

$$
\Lambda=\left[\begin{array}{cc}
|x|^{\alpha} & 0 \\
0 & |x|^{-\alpha}
\end{array}\right],
$$

and

$$
U=\left[\begin{array}{cc}
\cos |x|^{\beta} & \sin |x|^{\beta} \\
-\sin |x|^{\beta} & \cos |x|^{\beta}
\end{array}\right] .
$$

Then $U^{*} \Lambda U$ is a good weight for any of the operators discussed if and only if $\beta \geqslant \alpha$, which is exactly the condition called for by Theorem 5.1 of [1].

\section{REFERENCES}

1. S. Bloom, Weighted norm inequalities for vector-valued functions, Ph. D. dissertation, Washington University, St. Louis, Missouri, 1981.

2. R. R. Coifman and C. Fefferman, Weighted norm inequalities for maximal functions and singular integrals, Studia Math. 51 (1974), 241-250.

3. R. R. Coifman, R. Rochberg and G. Weiss, Factorization theorems for Hardy spaces in several variables, Ann. of Math. 103 (1976), 611-635.

4. H. Helson and G. Szegö, A problem in prediction theory, Ann. Math. Pura. Appl. 51 (1960), $107-138$.

5. R. A. Hunt, B. Muckenhoupt and R. L. Wheeden, Weighted norm inqualities for the conjugate function and Hilbert transform, Trans. Amer. Math. Soc. 176 (1973), 227-251.

6. E. Lotkowski and R. L. Wheeden, The equivalence of various Lipschitz conditions on the weighted oscillation of a function, Proc. Amer. Math. Soc. 61 (1976), 323-328.

7. B. Muckenhoupt, The equivalence of two conditions for weights, Studia Math. 49 (1974), 101-106.

8. Weighted norm inequalities for the Hardy maximal function, Trans. Amer. Math. Soc. 165 (1972), 207-226.

9. H. Pousson, Systems of Toeplitz operators on $H^{2}$. I, Proc. Amer. Math. Soc. 19 (1968), 603-608; II, Trans. Amer. Math. Soc. 133 (1968), 527-536.

10. R. Rabindranathan, On the inversion of Töeplitz operators, J. Math. Mech. 19 (1969), 195-206.

11. A. Cordoba and C. Fefferman, $A$ weighted norm inequality for singular integrals, Studia Math. 57 (1976), 97-101.

Department of Mathematics, Siena College, Loudonville, New York 12211 